\title{
REGULADOR NO BENEVOLENTE Y ASIMETRIAS DE INFORMACION. APROXIMACION CON UN MODELO DE AGENCIA
}

\author{
AN AGENCY MODEL FOR NON-BENEVOLENT REGULATION UNDER \\ ASYMMETRIC INFORMATION
}

\section{LOURDES MARCELA ESPINOZA VASQUEZ*}

Universidad Católica Boliviana

\section{RAUL RUBIN DE CELIS CEDRO**}

Universidad Católica Boliviana

\begin{abstract}
This document presents a theoretical model of a non-benevolent regulator and cost heterogeneous operators where the objective function to be maximized, besides considering consumers' and producers' surpluses, includes rent seeking from at least one of the operators that directly benefits the regulator. Under these conditions, the resulting regulatory contract complies with individual rationality and incentive compatibility; however, it introduces distortions that affect the most efficient operators resulting in costs to society as a whole. The main results from this model point towards the need to evaluate the relevance of maintaining regulation under such settings.
\end{abstract}

Keywords: Benevolent regulator, optimal prices, information asymmetries, incentive compatibility, participation constraint.

JEL Classification: $L 51, D 8, D 86$.

\section{Resumen}

El documento presenta un modelo teórico de un regulador no benevolente con agentes de diferente tipo, donde la función objetivo a maximizar, además

* Dra. en Economía, Profesora de la carrera de Economía. Email: lespinozav@ucb.edu.bo.

** Magíster en Economía, Profesor de la carrera de Economía. Email: r.rubindecelis@acad.ucb.edu.bo. 
de considerar el excedente del consumidor y del productor, incluye la extracción de rentas a alguno de los operadores que favorecen directamente al regulador. Se concluye que el contrato regulatorio resultante cumple con racionalidad individual y compatibilidad de incentivos, sin embargo, se introducen distorsiones que afectan a las empresas más eficientes generando costos para la sociedad en su conjunto. Los resultados sugieren la necesidad de evaluar la pertinencia de mantener la regulación bajo el esquema regulatorio planteado.

Palabras clave: Regulador benevolente, tarifas óptimas, asimetrías de información, compatibilidad de incentivos, restricción de participación.

Clasificación JEL: L51, D82, D86.

\section{INTRODUCCION}

Bajo un enfoque de la "Teoría del Interés Público"1 (Pigou (1932) y Bonbright (1961)), un regulador buscará maximizar el nivel de bienestar de la sociedad, al enfrentar una falla de mercado traducida en la presencia de un monopolio; esta maximización se logrará mediante la fijación de una tarifa óptima compatible con la asignación eficiente de recursos. Lo anterior supone que el regulador es "benevolente" 2 y que bajo un escenario de información simétrica, entre regulador y firma, se establece un contrato regulatorio óptimo en materia tarifaria.

Si bien el escenario de regulador benevolente sin asimetrías de información se convierte en un modelo básico para encontrar resultados óptimos, en cuanto a eficiencia asignativa y máximo bienestar, la evidencia empírica muestra que la regulación económica se caracteriza por la presencia de asimetrías de información entre regulado y regulador. Estas asimetrías pueden reflejarse por la presencia de selección adversa (información oculta) o riesgo moral (acción oculta), que llevan a un regulador a diseñar mecanismos que, plasmados en un contrato regulatorio (menú de tarifas), incentiven a la firma a revelar su verdadera información además de alinear su conducta con la de un regulador maximizador del bienestar social.

La inclusión dentro de la teoría regulatoria de escenarios que contemplan asimetrías de información dio lugar a una nueva línea de estudio denominada "Regulación por Incentivos" (Laffont y Tirole (1999) y Armstrong y Sappington (2005)), que tiene un fundamento en el diseño de mecanismos orientados a corregir

1 La teoría del interés público sostiene que la regulación surge en respuesta a la demanda de la sociedad por corregir ineficiencias del mercado y asume que la regulación beneficia a la sociedad en su conjunto buscando maximizar el bienestar de los consumidores y productores. Los promotores de esta línea defienden el reemplazo de la competencia por la participación estatal, fijando ya sea precios o bien controlando las condiciones de calidad y condiciones de servicio.

2 Bajo el enfoque de la Economía del Bienestar, un regulador benevolente es aquel que propicia los intereses de la sociedad y no los suyos, por tanto, posee como función objetivo la maximización del bienestar. 
o minimizar los problemas que surgen de la asimetría de información en mercados regulados. En materia regulatoria, la presencia de riesgo moral implica que variables endógenas (como el esfuerzo por reducir costos) no son observadas por el regulador ni siquiera por medio de auditorías regulatorias, mientras que con selección adversa es la firma la que posee mayor información respecto de variables exógenas (como la tecnología o más propiamente la función de costos). Considerando esos aspectos, la regulación es aproximada como un problema de agencia (Ross, 1973), donde el regulador (principal) busca maximizar el nivel de bienestar de la sociedad y el regulado (agente) tiene los incentivos correctos para trabajar con el mayor esfuerzo y revelar su tecnología ${ }^{3}$. Además de las restricciones informacionales que enfrenta el regulador al momento de establecer un contrato regulatorio, este modelo supone que se diseña correctamente el mecanismo con el que se maximiza la función de bienestar social, sujeta a las restricciones de racionalidad individual y compatibilidad de incentivos, encontrando resultados donde, aun con la presencia de asimetrías de información, el contrato regulatorio es óptimo.

Por su parte la "Teoría del Interés Privado" (Stigler, 1971; Posner, 1974 y Peltzman, 1976) sostiene que, desde el momento en que el regulador no tiene suficiente información para realizar su labor, enfrenta la posibilidad de que diferentes agentes en el proceso regulatorio (consumidores o firmas) traten de capturarlo para que maximice su propio interés. Debido a ese escenario la regulación beneficiaría a grupos de interés que capturan al regulador y que se encuentran favorecidos y protegidos por los reguladores ${ }^{4}$. Se asume que los agentes con mayor capacidad para realizar la captura del regulador son las firmas, tanto por la relación directa que tienen con los reguladores así como por la capacidad de pago que poseen.

Si bien los modelos teóricos desarrollados hasta el momento contemplan diversas situaciones en el proceso regulatorio enfocados en la presencia de asimetrías de información y el hecho de que el regulador sea o no benevolente, un aspecto no abordado por la teoría económica es aquella de un regulador no benevolente que ante la presencia de agentes heterogéneos en costos desea extraer rentas de alguno de los operadores regulados para su propio beneficio. Esta extracción de rentas puede ser interpretada como una situación en la que el regulador no cumple una característica esencial para su funcionamiento, tal cual es la "independencia" en la toma de sus decisiones respondiendo a intereses del hacedor de política, o bien posee algún sesgo por alguno de los operadores en función al tipo de propiedad (público o privado) que este puede presentar (Vickers y Yarrow, 1988), o finalmente por la presencia de corrupción, que según Laffont (2005) se encuentra presente fundamentalmente en economías en vías de desarrollo.

3 Dentro del estudio de la economía muchos de los temas que implican el diseño de mecanismos son explicados por los modelos de agencia, donde un principal posee una función objetivo que es alcanzable con el trabajo de un agente, quien incurre en un esfuerzo al trabajar para el principal.

4 Según señala Stigler (1971), bajo su enfoque "el regulador no es un agente benevolente, con conocimiento perfecto del mercado sino más bien es una organización que persigue su interés personal y se encuentra capturado por grupos de interés. Por tanto la regulación es obtenida por la industria, diseñada y operada para su propio beneficio". 
Bajo esta última idea, en el presente documento se desarrolla un modelo teórico de un regulador no benevolente que enfrenta operadores de diferente tipo según sus costos, tratando de extraer rentas de alguno de ellos para su propio beneficio, generando por tanto resultados ineficientes en cuanto al contrato regulatorio que se establece, aun cuando este cumple con las restricciones de racionalidad individual y compatibilidad de incentivos. Las distorsiones generadas son asumidas por las empresas más eficientes del mercado introduciendo costos para la sociedad en su conjunto. Los resultados sugieren la necesidad de evaluar la pertinencia de mantener la regulación bajo este esquema.

En tal sentido, en la siguiente sección se parte mostrando dos modelos básicos que suponen la presencia de un regulador benevolente y sin asimetrías de información, uno aportado por Baron y Myerson (1982) y el otro por Laffont y Tirole (1999), este último incluye el financiamiento de la labor regulatoria, mostrando el gasto en que incurren los consumidores por esta actividad. De ambos modelos se concluye que el menú de tarifas corresponde a una situación de primer mejor que hace máximo el nivel de bienestar social. En la Sección 3 se desarrolla el modelo de Laffont y Tirole (op. cit.) con asimetrías de información, obteniendo un contrato regulatorio óptimo, cuando la función objetivo de bienestar social se maximiza considerando las restricciones de racionalidad individual y compatibilidad de incentivos. En la Sección 4 se contrastan esos resultados con un escenario que describe a un regulador que deja de ser benevolente, enfrenta asimetrías de información y pretende extraer rentas de alguno de los operadores del mercado, encontrando un contrato regulatorio que, si bien cumple con las restricciones de compatibilidad de incentivos y racionalidad individual, es distorsionante debido a que incorpora costos para las firmas más eficientes. La Sección 5 presenta una comparación de escenarios con el fin de contrastar los resultados que se tienen en materia de eficiencia y maximización de bienestar para finalmente en la Sección 6 presentar las principales conclusiones del trabajo.

\section{MODELO BASICO: INFORMACION COMPLETA Y REGULADOR BENEVOLENTE}

De acuerdo con el planteamiento de Baron y Myerson (1982), se asume que la función objetivo del regulador es el bienestar de la sociedad, la que bajo un enfoque de mercados es la sumatoria del excedente del consumidor y del productor (este último aproximado mediante la función de beneficios (ver también Shy, 1995). Dicha función (que en general se asume como regulación de utilities $^{5}$ ) adopta la siguiente forma:

$$
W(q)=V(q)+\mu(P(q) q-c q-\rho)
$$

donde :

$V(q)=$ Excedente del consumidor que puede ser expresado como: $V(q)=\int P(q) d q-P(q) q$

5 Se denominan utilities a los servicios de utilidad pública, entre los que figuran servicios de agua potable por red, alcantarillado, telecomunicaciones, transportes, electricidad, gas por redes. 
$(P(q) q-c q-\rho)=$ Beneficio de la firma (ingresos menos costos totales)

$q=$ Cantidad producida del bien regulado

$\mu=$ Ponderador que establece el regulador respecto de los intereses de las firmas y los consumidores. Cuando $\mu=1$ se considera que el regulador privilegia con el mismo peso los intereses de firmas y usuarios, en general se asume que $0<\mu \leq 1$

Considerando la expresión del excedente del consumidor, el problema que debe resolver el regulador es el siguiente:

$$
\max _{q} W(q)=\int P(q) d q-P(q) q+\mu(P(q) q-c q-\rho)
$$

De las condiciones de primer orden respecto de $q$ se obtiene:

$$
\frac{P(q)-c}{P(q)}=\frac{1-\mu}{\mu} \frac{1}{\varepsilon}
$$

donde $\varepsilon=$ Elasticidad precio de la demanda.

Cuando el regulador pondera con el mismo peso los intereses de firmas y consumidores se cumple que $\mu=1$, por lo que a partir de la ecuación (2) y ante la presencia de información perfecta se concluye que el precio es igual al costo marginal, alcanzándose por tanto una situación de primer mejor donde se hace máximo el nivel de bienestar de la sociedad.

En presencia de monopolios naturales ${ }^{6}$, como el que caracteriza la regulación de los utilities, el resultado obtenido anteriormente debe ser entendido como una regulación de primer mejor que puede ser acompañada con la presencia de subsidios o bien de un esquema tarifario de precios no lineales, como tarifas en dos partes, donde el componente variable podría fijarse respecto del Costo Marginal de producción y el cargo fijo establecerse en función a la pérdida que genera la presencia de altos costos hundidos.

A partir de ese planteamiento básico, el modelo de regulador benevolente con información completa desarrollado por Laffont y Tirole (1999) incluye el esfuerzo que realiza la firma por disminuir costos, así como el financiamiento de un proceso regulatorio, que en la literatura económica se refleja como el costo de recaudar fondos de los contribuyentes (ya sea que venga de un impuesto o bien de una tasa cobrada a las firmas). Se considera por tanto un parámetro $\lambda$, exógeno a la firma regulada y

6 Los monopolios naturales son definidos como aquellas estructuras de mercado que presentan subaditividad de costos en un rango relevante de producción (Baumol, Panzar y Willig, 1982). Esta definición se aplica tanto a monopolios uniproducto como multiproducto. Bajo la teoría del interés público la Regulación Económica se justifica por la presencia de fallas de mercado, en este caso, por la presencia de monopolios naturales. 
que refleja el costo social de los fondos públicos ${ }^{7}$, una pérdida de peso muerto para los contribuyentes, ya que el cobro de impuestos puede generar distorsiones en la actividad productiva al reducir niveles de esfuerzo eficientes o bien inducir a realizar esfuerzos no productivos para evadir impuestos. Se asume que el bienestar de los consumidores disminuye en $(1+\lambda)$ pesos por cada peso de ingresos que por concepto de impuestos recauda el gobierno ${ }^{8}$.

Con información perfecta, el regulador observa el esfuerzo de la firma y desea incentivar este último para que sea más eficiente, es decir, busca bajar su nivel de costos. Para tal efecto y siguiendo lo planteado por Laffont y Tirole (op. cit.), se parte modelando una situación donde la firma regulada posee una función de costos de la forma:

$$
C=(\beta-e) q+\rho
$$

donde:

$\beta=$ Parámetro tecnológico (parámetro de eficiencia de la firma)

$e=$ Nivel de esfuerzo de la firma por reducir costos

$q=$ Cantidad producida del bien regulado

$\rho=$ Costo fijo conocido

El regulador observa el costo total y el producto, asimismo, se asume que realizar un esfuerzo por bajar el nivel de costo le genera a la firma una desutilidad igual a $\varphi(e)$, donde a mayor esfuerzo por bajar costos, mayor la desutilidad cumpliéndose que $\varphi^{\prime}(e)>0$. A su vez la tasa a la que crece dicho esfuerzo es creciente cumpliéndose que $\varphi^{\prime \prime}(e)>0$ (donde la función de esfuerzo es convexa), se asume también que $\varphi^{\prime \prime \prime}(e) \geq 0$, que refleja la condición suficiente para que el problema de optimización del regulador sea cóncavo. No olvidemos que bajo el escenario de información perfecta el regulador conoce la tecnología así como el nivel de esfuerzo que realiza la empresa.

El nivel de utilidad de la firma (excedente del productor) será:

$$
U=t-\varphi(e)
$$

donde $t=$ Transferencia neta ${ }^{9}$ que da el regulador a la firma (ingreso que dependerá del precio que le fije el regulador) y donde el agente se ocupa de la transferencia neta así como del esfuerzo.

7 En parte de la literatura acerca de regulación económica, planteada por Baron y Myerson (op. cit.), no se considera el parámetro $\lambda$, sino más bien un parámetro $\mu$, último que pondera los intereses de las firmas dentro de la función objetivo del regulador.

8 En el presente documento se asume un $\lambda>0$, ya que se pretende capturar el impacto que tiene el financiamiento del proceso regulatorio sobre los consumidores que demandan bienes o servicios de sectores regulados.

9 Se define como transferencia neta porque los costos serán reembolsados por el regulador. 
Bajo información completa, el regulador busca garantizar un beneficio normal que será atractivo para que la firma, trabajando con un nivel de esfuerzo óptimoXX, ingrese al mercado cumpliendo así la condición de racionalidad individual o restricción de participación ${ }^{10}$.

Para los consumidores su excedente neto puede ser expresado de la siguiente forma:

$$
E C=V(q)-(1+\lambda)(t+C)
$$

donde:

$V(q)=$ Excedente bruto de los consumidores

$(t+C)=$ Transferencia a la empresa más el costo total de producción de la firma

$\lambda=$ Costo sombra de los fondos públicos

$(1+\lambda)=$ Desutilidad generada por los impuestos distorsionantes que se aplican para financiar la regulación, que son asumidos por los contribuyentes.

Operando en la ecuación (4) se obtiene la siguiente expresión:

$$
E C=V(q)-(1+\lambda)(t+(\beta-e) q+\rho)
$$

Por lo que la función objetivo de bienestar para un regulador utilitarista será la siguiente:

$$
W=V(q)-(1+\lambda)[t+(\beta-e) q+\rho]+[t-\varphi(e)]
$$

Con el fin de tener una expresión del bienestar que refleje la utilidad de la firma podemos reescribir la ecuación (5) de la siguiente manera:

$$
W=V(q)-(1+\lambda)[t+(\beta-e) q+\rho]+[t-\varphi(e)]+\lambda[t-\varphi(e)]-\lambda[t-\varphi(e)]
$$

Por lo que bajo un modelo simple, con información completa el problema que debe resolver el regulador es el siguiente:

$$
\max _{(q, e)} W=V(q)-(1+\lambda)[(\beta-e) q+\rho+\varphi(e)]-\lambda U
$$

Con información perfecta el regulador busca que $U=0 \mathrm{o} t=\varphi(e)$, por lo que las condiciones de optimalidad que se obtienen son:

10 La restricción de participación o de racionalidad individual nos dice que un agente, condicionado a su tipo $\delta$, está dispuesto a participar en una determinada transacción o intercambio si se cumple que $U(\delta) \geq 0, \forall \delta \in(\underline{\delta}, \bar{\delta})$. 


$$
\begin{aligned}
& \frac{d W}{d e}=(1+\lambda)\left[\frac{d \varphi(e)}{d e}-q\right]=0 \text { o } \frac{d \varphi(e)}{d e}=q \\
& \frac{d W}{d q}=\frac{d V(q)}{d q}-(1+\lambda)(\beta-e)=0 \text { о } \frac{d V(q)}{d q}=(1+\lambda)(\beta-e)
\end{aligned}
$$

Las condiciones de optimalidad anteriormente encontradas deben reflejarse en la formulación de un contrato regulatorio, que puede ser presentado en diferentes menús de tarifas ${ }^{11}$ que logren autorrevelación de información por parte de las empresas al regulador. Dicho menú puede ser escrito de forma general como $t=t(\beta, C)$, que al ser linealizado 12 toma la forma $t(\beta, C)=t^{*}\left(\beta, C^{*}\right)+t^{\prime}\left(\beta, C^{*}\right)\left(\mathrm{C}-C^{*}\right)(6)$, donde $\left(\mathrm{C}-C^{*}\right)$ muestra la diferencial de costos de un nivel efectivo $(\mathrm{C})$ respecto de uno óptimo $\left(C^{*}\right)$. Por otra parte, considerando que $t=U+\varphi(\beta-C)$, tenemos que $b=\frac{d t}{d C}=-\frac{d \varphi(\beta-C)}{d(\beta-C)}=\varphi^{\prime}\left(e^{*}(\beta)\right)$ y donde $a(\beta)=t^{*}(\beta)+\varphi^{\prime}\left(e^{*}(\beta)\right) C^{*}(\beta)$. Operando en la ecuación (6) la expresión final de la tarifa será:

$$
t(C)=a-b C
$$

De la ecuación (7) destaca que $b$ refleja la fracción de costos que cubre la firma, por lo que puede ser visto como el poder que el esquema de incentivos tiene sobre la tarifa. El contrato será de alto o bajo poder en función a los incentivos que se da a la firma para que realice o no un mayor esfuerzo por disminuir sus $\operatorname{costos}^{13}$. Un contrato será de bajo poder si $b=0$, es decir, uno en el que se retribuya a la firma exactamente en función a los costos declarados por la misma. Por otra parte cuando $b=1$ se tendrá un contrato tarifario donde $t=t *(\beta)$, es decir, uno en el que el operador alcance el nivel de costos óptimos y por tanto sea de alto poder. Finalmente, en la medida en la que $0<b<1$ se tendrá un esquema híbrido entre un contrato de alto y otro de bajo poder.

En el caso de un regulador benevolente con información perfecta el nivel de costo así como el esfuerzo que la firma debe realizar para disminuir los mismos se observan directamente, en tal sentido $b=1$, por lo que, a partir de las condiciones de optimalidad anteriormente encontradas, se inducirá a la firma a que trabaje con un nivel de costo óptimo y por tanto la fijación tarifaria será óptima generando un contrato regulatorio de alto poder.

Del modelo de regulador benevolente con información perfecta, con y sin financiamiento de la labor regulatoria, se tienen las siguientes conclusiones:

11 La teoría sobre discriminación de precios supone que el diseño óptimo de un menú de tarifas logra autoselección y autorrevelación de información.

12 Dicha expresión se linealiza empleando una serie de Taylor de orden uno.

13 Se definen contratos de alto poder aquellos esquemas tarifarios que inducen a mayor esfuerzo por parte del operador para bajar el nivel de costo y por tanto tener una tarifa óptima, por su parte los contratos de bajo poder no contienen incentivos para disminuir costos y por tanto las tarifas tienden a ser no óptimas. 
a) El contrato regulatorio es uno de alto poder, donde la tarifa se fija de acuerdo con el nivel de costo óptimo (primer mejor), que garantiza la maximización del bienestar social.

b) La situación de primer mejor, en la regulación de monopolios naturales, es entendida como la fijación de una tarifa no lineal, donde la pérdida que se genera por la presencia de altos costos hundidos puede ser cubierta con un cargo fijo o bien con la introducción de un subsidio.

\section{INFORMACION INCOMPLETA Y REGULADOR BENEVOLENTE}

Definida la tarifa óptima que debería cobrar un regulador a un monopolista en un escenario donde no existe asimetría de información y la función objetivo a maximizar es el bienestar social, se considera ahora una situación donde el regulador no puede observar la tecnología, ni el nivel de esfuerzo por parte de la firma para bajar costos. Dentro de este escenario el regulador enfrenta problemas tanto de selección adversa (desconocimiento de la tecnología $(\beta)$ o costos) como de riesgo moral (esfuerzo $(e)$ no observable), constituyéndose la tarifa en el resultado del mecanismo (incentivo) que lleva a las firmas a revelar su tecnología y las induce a realizar el mayor esfuerzo por disminuir sus costos.

Esta modelación considera la presencia de un regulador que enfrenta dos posibles tipos de operadores, uno eficiente y otro ineficiente. El regulador sabe que con probabilidad $(1-v)$ puede enfrentar un operador que trabaja con una tecnología $\bar{\beta}$ (ineficiente), y con probabilidad $v$ un operador que tiene una tecnología $\underline{\beta}$ (eficiente) ${ }^{14}$.

El resultado óptimo que debe alcanzar el regulador es que mediante la tarifa, el operador desee ingresar al mercado y se revele como lo que efectivamente es, esto último quiere decir que en su proceso de maximización del bienestar debe lograr una compatibilidad de incentivos, de tal forma que la mejor respuesta de cada uno de los operadores sea decir la verdad ${ }^{15}$. La restricción de compatibilidad de incentivos considera las transferencias o rentas que se dan a las firmas, de tal forma que se formula para cada uno de los tipos de agentes, con el fin de que se revelen como lo que efectivamente son. Diseñada la tarifa, el contrato tarifario se aplicará finalmente a un monopolio, que será el que ingresará al mercado a proveer el bien o servicio.

Bajo los supuestos de un regulador benevolente y dos tipos de agentes, se parte modelando la función objetivo del regulador. Se asume que la firma más eficiente es aquella cuyo esfuerzo por disminuir costos es más bajo y que la firma más ineficiente debe realizar un mayor esfuerzo para tener ganancias en eficiencia, Spence (1973).

14 Se asume que la probabilidad está bien definida y por tanto se cumple que $0<v<1$ y $\sum_{i=1}^{2} v_{i}=1$. Asimismo, debido a que el objeto del documento es diseñar un mecanismo de revelación, se asumen conocidas las probabilidades de que la firma sea o no eficiente.

15 El cumplimiento de la compatibilidad de incentivos caracteriza un equilibrio de Nash, que fija el principal (regulador) al agente (regulado). 
Debido a una probabilidad de que el regulador pueda enfrentar agentes de un tipo o del otro, la función de bienestar de la sociedad puede ser escrita como una función Von Neumann-Mongerstern (ver Jehle y Reny (2011)) de la siguiente forma:

$$
\begin{gathered}
\max _{(\bar{q}, \underline{q}, \bar{e}, \underline{e})} W=v[V(\underline{q})-(1+\lambda)((\underline{\beta}-\underline{e}) \underline{q}+\rho+\varphi(\underline{e}))-\lambda \underline{U}] \\
\quad+(1-v)[V(\bar{q})-(1+\lambda)((\bar{\beta}-\bar{e}) \bar{q}+\rho+\varphi(\bar{e}))-\lambda \bar{U}]
\end{gathered}
$$

En este escenario la empresa ineficiente recibe beneficios normales y no tiene incentivos a declararse como eficiente, ya que reduce la posibilidad de capturar alguna renta porque, en términos relativos, de revelarse como eficiente recibiría una transferencia más baja como resultado de mostrarse como una firma de costos bajos. Para la firma ineficiente la restricción de participación se expresa como $\bar{U}=t(\bar{\beta})-\varphi(\bar{\beta}-\bar{c})=0$, mientras que la de compatibilidad de incentivos se cumplirá si:

$$
t(\bar{\beta})-\varphi(\bar{\beta}-\bar{c}) \geq t(\underline{\beta})-\varphi(\bar{\beta}-\underline{c})
$$

Es decir, a una firma de costos altos le conviene revelarse como tal antes que declararse como una firma de costos bajos, ya que de hacerse pasar por una firma eficiente la transferencia que obtendría sería menor, obteniendo pérdidas por decir que es eficiente y contradiciendo su restricción de participación como firma ineficiente.

En el caso de la empresa eficiente, y considerando que bajo este esquema regulatorio se contempla reembolso de costos, la restricción de compatibilidad de incentivos se la plantea de la siguiente forma:

$$
\underline{U}>t(\bar{\beta})-\varphi(\bar{\beta}-\bar{c})+\varphi(\bar{\beta}-\bar{c})-\varphi(\underline{\beta}-\bar{c})
$$

Si se considera que un regulador buscará hacer sostenible la operación de la firma en el mercado y no dejarle beneficios extraordinarios, la anterior condición se la asume con igualdad estricta. Realizando algunas operaciones dicha restricción puede ser expresada como:

$$
\begin{gathered}
\underline{U}=t(\bar{\beta})-\varphi(\bar{\beta}-\bar{c})+\varphi(\bar{\beta}-\bar{c})-\varphi(\underline{\beta}-\bar{c}) \\
\underline{U}=\bar{U}+\varphi(\bar{e})-\varphi(\underline{\beta}+\bar{\beta}-\bar{\beta}-\bar{c}) \\
\underline{U}=\bar{U}+\varphi(\bar{e})-\varphi(\bar{e}-\Delta \beta)
\end{gathered}
$$

Teniendo en cuenta que $\bar{U}=0, \underline{U}=\phi(\bar{e})$, donde $\phi(\bar{e})=\varphi(\bar{e})-\varphi(\bar{e}-\Delta \beta)$, que se constituye en la renta informacional ${ }^{16}$. Esta renta informacional garantiza que

16 Se denomina renta informacional a la disposición a pagar que algunos agentes económicos poseen para que los demás se revelen con su verdadera información 
el eficiente no se desvíe de declarar la verdad para obtener beneficios del engaño (declararse como ineficiente y tener la transferencia del ineficiente que es más alta debido a sus costos altos), la función $\phi(\bar{e})$ es convexa, por lo que $\underline{U} \geq 0$, de donde la restricción de participación de la firma eficiente se expresará como $t(\underline{\beta})-\varphi(\underline{\beta}-\underline{c}) \geq 0$.

Incluyendo las restricciones de participación y compatibilidad de incentivos en la ecuación (8), la función a maximizar por el regulador es:

$$
\begin{gathered}
\max _{(\bar{q}, \underline{q}, \bar{e}, \underline{e})} W=v[V(\underline{q})-(1+\lambda)((\underline{\beta}-\underline{e}) \underline{q}+\rho+\varphi(\underline{e}))+\lambda \phi(\bar{e})] \\
+(1-v)[V(\bar{q})-(1+\lambda)((\bar{\beta}-\bar{e}) \bar{q}+\rho+\varphi(\bar{e}))]
\end{gathered}
$$

Las condiciones de primer orden serán por lo tanto:

$$
\begin{aligned}
& \frac{d W}{d \underline{q}}=v \frac{d V(\underline{q})}{d \underline{q}}-v(1+\lambda)(\underline{\beta}-\underline{e})=0 \\
& \text { de donde: } \frac{d V(\underline{q})}{d \underline{q}}=(1+\lambda)(\underline{\beta}-\underline{e}) \\
& \frac{d W}{d \bar{q}}=(1-v) \frac{d V(\bar{q})}{d \bar{q}}-(1-v)(1+\lambda)(\bar{\beta}-\bar{e})=0 \\
& \text { de donde: } \frac{d V(\bar{q})}{d \bar{q}}=(1+\lambda)(\bar{\beta}-\bar{e}) \\
& \frac{d W}{d \underline{e}}=(1+\lambda)\left[\frac{d \varphi(e)}{d \underline{e}}-\underline{q}\right]=0 \text { o } \frac{d \varphi(\underline{e})}{d \underline{e}}=\underline{q} \\
& \frac{d W}{d \bar{e}}=-v \lambda \frac{d \phi(\bar{e})}{d(\bar{e})}-(1-v)\left((1+\lambda)-\bar{q}+\frac{d \varphi(\bar{e})}{d(\bar{e})}\right)=0 \\
& \text { o } \frac{d \varphi(\bar{e})}{d(\bar{e})}=\bar{q}-\frac{v}{1-v} \quad \frac{\lambda}{1+\lambda} \frac{d \phi(\bar{e})}{d(\bar{e})}
\end{aligned}
$$

Con la presencia de información asimétrica se debe diseñar un contrato regulatorio que induzca al operador a decir la verdad, el menú de contratos que se refleja en una tarifa no lineal, expresada genéricamente en la ecuación (7), debe inducir a la firma a decir la verdad respecto de $(\beta)$ e incentivarla a realizar un esfuerzo óptimo $\left(e^{*}\right)$. A partir de las condiciones de primer orden, obtenidas anteriormente, se demuestra que bajo este escenario la tarifa se constituirá en un mecanismo que logre que la firma revele su tecnología y realice su mayor esfuerzo.

La demostración de cómo se resuelven los problemas de selección adversa y riesgo moral con el contrato diseñado son las que a continuación se presentan. Se 
parte del siguiente problema de optimización que contempla maximizar la utilidad de la firma respecto de $e$ y $\beta$.

$$
\max _{(e, \beta)} U=t^{*}(\hat{\beta})+\varphi^{\prime}\left(e^{*}(\hat{\beta})\right)\left(\beta-e-\hat{\beta}+e^{*}\right)-\varphi(e)
$$

de las condiciones de primer orden $\frac{d U}{d e}$ se tiene que: $\varphi^{\prime}\left(e^{*}\right)=\varphi^{\prime}(e)$ por lo que $e^{*}(\hat{\beta})=e$, resolviendo así el tema de riesgo moral, para demostrar que se resuelve el problema de selección adversa se asume dos tipos de tecnología $\beta, \beta^{\prime} \epsilon[\beta, \bar{\beta}]$, a partir de la restricción de compatibilidad de incentivos se cumple que: $t(\beta)-\varphi(\beta-C(\beta)) \geq t\left(\beta^{\prime}\right)-\varphi\left(\beta-C\left(\beta^{\prime}\right)\right)$ y $t\left(\beta^{\prime}\right)-\varphi\left(\beta^{\prime}-C\left(\beta^{\prime}\right)\right) \geq t(\beta)-\varphi\left(\beta^{\prime}-C(\beta)\right)$, reordenando la anterior expresión se llega a:

$$
\frac{\varphi\left(\beta-C\left(\beta^{\prime}\right)\right)-\varphi(\beta-C(\beta))}{\beta^{\prime}-\beta}=\frac{t\left(\beta^{\prime}\right)-t(\beta)}{\beta^{\prime}-\beta}=\frac{\varphi\left(\beta^{\prime}-C\left(\beta^{\prime}\right)\right)-\varphi\left(\beta^{\prime}-C(\beta)\right)}{\beta^{\prime}-\beta}
$$

evaluando esa expresión en sus límites nos queda que: $\frac{d t}{d \beta}=-\frac{d \varphi(e)}{d e} \frac{d C}{d \beta}$, lo que es equivalente a: $\frac{d t}{d \beta}+\frac{d \varphi(e)}{d e} \frac{d C}{d \beta}=0$

A partir de lo anterior y realizando algunas operaciones se llega a:

$$
\begin{aligned}
& \frac{d t^{*}}{d \beta}-\varphi^{\prime}\left(e^{*}(\beta)\right)\left[-1+\frac{d e^{*}(\hat{\beta})}{d \hat{\beta}}\right]-\varphi^{\prime \prime}\left(e ^ { * } ( \beta ) \frac { d e ^ { * } ( \beta ) } { d \beta } \left[\left(\beta-\hat{\beta}-e-e^{*}(\hat{\beta})\right]=0\right.\right. \\
& \frac{d t^{*}(\beta)}{d \beta}+\varphi^{\prime}\left(e^{*}(\beta)\right)\left[\frac{d C(\beta)}{d \beta}\right]-\varphi^{\prime \prime}\left(e ^ { * } ( \beta ) \frac { d e ^ { * } ( \beta ) } { d \beta } \left[\left(\beta-\hat{\beta}-e-e^{*}(\hat{\beta})\right]=0\right.\right.
\end{aligned}
$$

de donde: $[\beta-\hat{\beta}]=0$ por lo que: $\beta=\hat{\beta}$ resolviendo el problema de selección adversa y logrando establecer si la empresa es eficiente o ineficiente, es decir, se alcanza un equilibrio separador, Spence (op. cit.).

Demostrado que el diseño de un menú de tarifas óptimo resuelve los problemas de asimetría de información, las tarifas óptimas se establecerán según tipo de operador, las que lograrán que cada empresa se revele de acuerdo con la tecnología que posee. Se tiene por tanto el siguiente menú de tarifas:

Empresa eficiente: $a(\underline{\beta})=\varphi(\underline{e})+\phi(\bar{e})+\varphi^{\prime}(\underline{e})(\underline{\beta}-\underline{e})$

$$
b(\underline{\beta})=\varphi^{\prime}(\underline{e})
$$

Empresa ineficiente: $a(\bar{\beta})=\varphi(\bar{e})+\varphi^{\prime}(\bar{e})(\bar{\beta}-\bar{e})$

$$
b(\bar{\beta})=\varphi^{\prime}(\bar{e})
$$


Considerando la ecuación (7) la expresión final de las tarifas será:

Empresa eficiente: $t^{*}(\underline{\beta})=\varphi(\underline{e})+\phi(\bar{e})$

Empresa ineficiente: $t^{*}(\bar{\beta})=\varphi(\bar{e})$

De lo anterior, los principales hallazgos bajo un escenario de información asimétrica y regulador benevolente son los siguientes:

a) Respecto de un escenario sin asimetrías de información este modelo incluye, dentro de su menú de tarifas, incentivos para la revelación de información por parte de la firma.

b) Se incurre en un costo de transacción para obtener información, tanto de la tecnología como del nivel de esfuerzo que realiza la firma por disminuir sus costos. Este costo se traduce en una renta informacional $(\phi(\bar{e}))$ que el regulador, mediante la tarifa, debe retribuir a la empresa eficiente, para que esta se revele como tal y se alcance un equilibrio separador.

c) El menú de tarifas establecido por el regulador será óptimo en la medida que el proceso de maximización del bienestar social incluya las restricciones de compatibilidad de incentivos y de racionalidad individual. El contrato regulatorio así diseñado resuelve los problemas de selección adversa y riesgo moral, remunerando correctamente a la firma, ya sea que esta sea eficiente o ineficiente.

\section{REGULACION CON ASIMETRIA DE INFORMACION Y REGULADOR NO BENEVOLENTE}

Los resultados encontrados bajo condiciones de regulador benevolente con y sin asimetrías de información, permite analizar las implicancias de un tercer escenario con la presencia de un regulador no benevolente que enfrenta asimetrías de información y que pretende obtener rentas de alguno de los operadores. En este caso, a diferencia de un modelo de captura, el regulador no busca propiciar los intereses de un grupo de interés determinado, sino que busca incrementar una transferencia que le sea de utilidad y que provenga de los operadores regulados. Bajo este escenario se modela el efecto que genera esta situación sobre las tarifas que finalmente son pagadas por los consumidores.

La función objetivo a maximizar por un regulador neutral al riesgo será:

$$
W=V(q)-(1+\lambda)(t+C)+t-\varphi(e)+\alpha \lambda(t-\varphi(e))
$$

donde:

$V(q)-(1+\lambda)(t+C)=$ Excedente neto de los consumidores

$t-\varphi(e)=$ Beneficios del monopolio 
$\alpha \lambda(t-\varphi(e))=$ Utilidad para el regulador, donde $\alpha$ refleja la proporción de los beneficios de la firma que van al regulador por tanto $\alpha \epsilon(0,1)$

Se asume la función de costo total del operador con un costo fijo y un costo variable de la forma $C=(\beta-e) q+\rho$. Definida así la función de costos, se puede reescribir la función (12), donde a través de los costos se captura el impacto del parámetro tecnológico y del nivel de esfuerzo de la firma. Se tiene por tanto:

$$
\begin{gathered}
W=V(q)-(1+\lambda)(t+C)-\lambda(t-\varphi(e))+(1+\lambda)(t-\varphi(e))+\lambda \alpha(t-\varphi(e)) \\
W=V(q)-(1+\lambda)(t+C-t+\varphi(e))+\lambda(\alpha-1)(t-\varphi(e)) \\
W=V(q)-(1+\lambda)(C+\varphi(e))-\lambda(1-\alpha)(t-\varphi(e))
\end{gathered}
$$

Obteniendo finalmente la expresión donde el regulador desea maximizar los intereses de la sociedad y sus propios intereses:

$$
W=V(q)-(1+\lambda)((\beta-e) q+\rho+\varphi(e))-\lambda(1-\alpha)(t-\varphi(e))
$$

La ecuación (13) es la función objetivo a ser maximizada por el regulador cuyos argumentos son $q$ y $e$. Se advierte que el esfuerzo de la firma determina la transferencia que finalmente obtiene el regulador para sí mismo.

Para ver los impactos del parámetro $\alpha$ sobre la función objetivo, se asumen dos escenarios, el primero contempla la presencia de información perfecta, el segundo incluye asimetría de información con dos tipos de agentes que no pueden ser diferenciados en cuanto a tecnología y esfuerzo, para finalizar con un modelo que considera un continuo de tipos, este último representa un modelo general en el que se admite la posibilidad de varios tipos de agentes según su tecnología, en este caso se asume que la fuente de la asimetría proviene de diferentes niveles de eficiencia que poseen una gama de operadores.

\section{Regulador no benevolente con información perfecta}

Partimos retomando la ecuación (13), en un escenario de información completa el regulador no dejará renta al monopolista, es decir, se cumplirá que $U=0$ por lo que $t=\varphi(e)$. En tal caso, es fácil deducir que el modelo se torna equivalente a uno donde el regulador es benevolente, es decir, donde se tiene una función objetivo a maximizar de la forma:

$$
\max _{(q, e,)} W=V(q)-(1+\lambda)((\beta-e) q+\rho+\varphi(e))
$$

donde las condiciones de optimalidad serán las encontradas anteriormente, expresadas de la siguiente forma: 


$$
\begin{aligned}
& \frac{d W}{d e}=(1+\lambda)\left[\frac{d \varphi(e)}{d e}-q\right]=0 \text { о } \frac{d \varphi(e)}{d e}=q \\
& \frac{d W}{d q}=\frac{d V(q)}{d q}-(1+\lambda)(\beta-e)=0
\end{aligned}
$$

En materia tarifaria el resultado de ese proceso de optimización muestra un menú de tarifas óptimo y eficiente. Una conclusión con información perfecta, es que bajo este escenario, un regulador no puede obtener ventajas cuando trata de extraer rentas a los operadores, lo anterior porque no solo el regulador conoce la función de producción de las firmas, sino que los operadores conocen la tecnología de sus rivales. Sin asimetrías de información cuando un regulador establece una tarifa, esta refleja la diferencia en costos que los operadores tienen, por lo que un operador, eficiente o ineficiente, no aceptará que el regulador quiera cobrarle rentas por algo que él ya conoce, en este escenario si se quiere extraer renta de alguno de los operadores estos pueden optar por no ingresar a este mercado que presenta una regulación asimétrica.

\section{Regulador no benevolente con dos tipos de agentes}

Bajo el supuesto de información asimétrica, con un regulador que busca rentas por parte de algunos de los operadores que son de diferente tipo, se retoma la función expresada en la ecuación (13). A causa de esa función el regulador sabe que la probabilidad de enfrentar un operador eficiente es igual a $v$ y de enfrentar un operador ineficiente es $(1-v)$, es decir, el regulador conoce la distribución de probabilidad de los operadores, pero no sabe quién es quién con certeza.

Bajo ese supuesto se plantea el problema del regulador como una función de bienestar esperada de la forma:

$$
\begin{gathered}
\max _{(\bar{q}, \underline{q}, \bar{e}, \underline{e})} W=v[V(\underline{q})-(1+\lambda)((\underline{\beta}-\underline{e}) \underline{q}+\rho+\varphi(\underline{e}))-\lambda(1-\alpha)(t(\underline{\beta})-\varphi(\underline{e}))] \\
\quad+(1-v)[V(\bar{q})-(1+\lambda)((\bar{\beta}-\bar{e}) \bar{q}+\rho+\varphi(\bar{e}))-\lambda(1-\alpha)(t(\bar{\beta})-\varphi(\bar{e}))]
\end{gathered}
$$

De acuerdo con lo establecido con anterioridad $t(\beta)-\varphi(e)=U$, por lo que la anterior expresión se puede reescribir como:

$$
\begin{gathered}
\max _{(\bar{q}, \underline{q}, \bar{e}, \underline{e})} W=v[V(\underline{q})-(1+\lambda)((\underline{\beta}-\underline{e}) \underline{q}+\rho+\varphi(\underline{e}))-\lambda(1-\alpha) \underline{\mathrm{U}}]+ \\
(1-v)[V(\bar{q})-(1+\lambda)((\bar{\beta}-\bar{e}) \bar{q}+\rho+\varphi(\bar{e}))-\lambda(1-\alpha) \overline{\mathrm{U}}]
\end{gathered}
$$

Asimismo y de acuerdo con los resultados presentados en la sección anterior, por restricción de compatibilidad de incentivos se tiene que $\underline{U}=\phi(\bar{e})$ y por su parte 
$\bar{U}=0$ por lo que finalmente se puede expresar la función objetivo a maximizar de la siguiente manera:

$$
\begin{gathered}
\max _{(\bar{q}, \underline{q}, \bar{e}, \underline{e})} W=v[V(\underline{q})-(1+\lambda)((\underline{\beta}-\underline{e}) \underline{q}+\rho+\varphi(\underline{e}))-\lambda(1-\alpha) \phi(\bar{e})]+ \\
(1-v)[V(\bar{q})-(1+\lambda)((\bar{\beta}-\bar{e}) \bar{q}+\rho+\varphi(\bar{e}))]
\end{gathered}
$$

A partir de la ecuación (14) las condiciones de primer orden que se obtienen son:

$$
\frac{d W}{d \underline{q}}=v \frac{d V(\underline{q})}{d \underline{q}}-v(1+\lambda)(\underline{\beta}-\underline{e})=0
$$

de donde:

$$
\begin{aligned}
& \frac{d V(\underline{q})}{d \underline{q}}=(1+\lambda)(\underline{\beta}-\underline{e}) \\
& \frac{d W}{d \underline{e}}=(1+\lambda)\left[\frac{d \varphi(\underline{e})}{d \underline{e}}-\underline{q}\right]=0 \text { o } \frac{d \varphi(\underline{e})}{d \underline{e}}=\underline{q} \\
& \frac{d W}{d \bar{q}}=(1-v) \frac{d V(\bar{q})}{d \bar{q}}-(1-v)(1+\lambda)(\bar{\beta}-\bar{e})=0
\end{aligned}
$$

de donde:

$$
\begin{aligned}
& \frac{d V(\bar{q})}{d \bar{q}}=(1+\lambda)(\bar{\beta}-\bar{e}) \\
& \frac{d W}{d \bar{e}}=-v \lambda(1-\alpha) \frac{d \phi(\bar{e})}{d(\bar{e})}-(1-v)(1+\lambda)\left(-\bar{q}+\frac{d \varphi(\bar{e})}{d(\bar{e})}\right)=0
\end{aligned}
$$

de donde:

$$
-\frac{v}{1-v} \frac{\lambda}{1+\lambda}(1-\alpha) \frac{d \phi(\bar{e})}{d(\bar{e})}=-\bar{q}+\frac{d \varphi(\bar{e})}{d(\bar{e})}
$$

De la anterior expresión destaca que el esfuerzo óptimo para la empresa ineficiente puede expresarse como:

$$
\frac{d \varphi(\bar{e})}{d(\bar{e})}=\bar{q}-\frac{v}{1-v} \frac{\lambda}{1+\lambda}(1-\alpha) \frac{d \phi(\bar{e})}{d(\bar{e})}
$$

Se advierte que las condiciones de optimalidad que se encuentran bajo este escenario son las mismas a las del modelo de regulador benevolente con asimetrías 
de información con excepción de la ecuación (15). Si se retoma la ecuación (7), se determina que las tarifas dentro de este modelo son las mismas a las obtenidas en las ecuaciones (10) y (11). Por tanto, el menú de tarifas será óptimo debido a que resuelve el problema de la asimetría de información tanto para el parámetro tecnológico como para el nivel de esfuerzo óptimo al que se induce a la firma para reducir sus costos.

Si bien el menú de tarifas encontrado es óptimo, debe considerarse dentro del mercado, el impacto que genera la presencia de un porcentaje de renta que espera el regulador. Para aproximar dicho impacto se realizan dos análisis, el primero donde se determina cómo el esfuerzo óptimo que realiza una firma se ve afectado por el porcentaje de renta que el regulador pretende obtener en una proporción expresada por $\alpha$, y el segundo determinando el impacto de $\alpha$ sobre la tarifa que se establece en el mercado.

Para determinar cómo el esfuerzo óptimo de una firma se ve afectado por $\alpha$, se parte de un análisis de estática comparativa considerando la condición de optimalidad que sale del nivel de bienestar respecto de los niveles de esfuerzo que realizan las firmas.

En el caso de la firma eficiente se tiene: $\frac{d W}{d e}=(1+\lambda)\left[\frac{d \varphi(\underline{e})}{d e}-\underline{q}\right]$ por lo que $\frac{d \underline{e}}{d \alpha}=0$, es decir, que $\alpha$ no afecta el nivel de esfuerzo que pueda realizar la empresa eficiente.

Para la firma ineficiente se retoma la expresión (15) y se la expresa como:

$$
\frac{d \varphi(\bar{e})}{d(\bar{e})}-\bar{q}+\frac{v}{1-v} \frac{\lambda}{1+\lambda}(1-\alpha) \frac{d \phi(\bar{e})}{d(\bar{e})}=0
$$

A partir del teorema de la función implícita se resuelve (16) obteniendo lo siguiente:

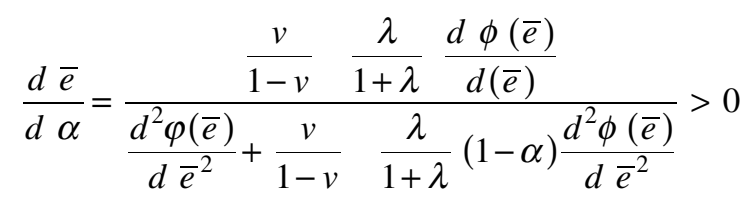

La anterior expresión confirma un aspecto que en materia de mecanismos se cumple, es decir, que el regulador debe exigir un alto nivel de esfuerzo al ineficiente. Sin embargo, se debe recordar que bajo este escenario el regulador desea obtener una renta expresada en $G=\lambda \alpha(\underline{t}-\varphi(\underline{e})$, la que de manera equivalente puede ser vista como $G=\lambda \alpha(\phi(\bar{e}))$, donde la renta del regulador depende a su vez de la renta informacional que se tiene que retribuir a la firma eficiente para que se revele como tal. Debido a que se encontró que $\frac{d \bar{e}}{d \alpha}>0$ se cumple que:

$$
\frac{d G}{d \alpha}=\lambda[\phi(\bar{e})]+\lambda \alpha\left[\frac{d \phi(\bar{e})}{d(\bar{e})} \frac{d \bar{e}}{d \alpha}\right]>0
$$

En otras palabras, la renta que espera obtener el regulador será más alta en la medida que mayor sea el porcentaje que espere extraer de renta a los operadores, última que a su vez depende de la tarifa que establezca el regulador. 
Para determinar el impacto de $\alpha$ sobre la tarifa se parte de lo siguiente:

$$
\frac{d t}{d \alpha}=\frac{d a(\beta)}{d \bar{e}} \frac{d \bar{e}}{d \alpha}-\frac{d b(\beta)}{d \bar{e}} \frac{d \bar{e}}{d \alpha} C=\left(\frac{d a(\beta)}{d \bar{e}}-\frac{d b(\beta)}{d \bar{e}} C\right) \frac{d \bar{e}}{d \alpha}
$$

cuando la firma es eficiente el efecto de $\alpha$ en la tarifa será:

$$
\frac{d t}{d \alpha}=\frac{d \phi(\bar{e})}{d \bar{e}} \frac{d \bar{e}}{d \alpha}>0
$$

cuando la firma es ineficiente el efecto de XX en la tarifa será:

$$
\frac{d t}{d \alpha}=\left[\varphi^{\prime}(\bar{e})+\varphi^{\prime \prime}(\bar{e})(\bar{\beta}-\bar{e})-\varphi^{\prime}(\bar{e})-\varphi^{\prime \prime}(\bar{e}) \bar{C}\right] \frac{d \bar{e}}{d \alpha}
$$

A causa de que el mecanismo hace que la firma se revele como lo que es, el efecto para la empresa ineficiente será nulo, es decir, se cumple que $\bar{C}=\bar{\beta}-\bar{e}$, es decir, $\frac{d t}{d \alpha}=0$.

En resumen, la extracción de rentas, por parte del regulador a los operadores regulados, tendrá impacto sobre la tarifa de la firma eficiente y no así en la ineficiente, determinando que para la eficiente sea más costoso revelarse y trabajar dentro del mercado como lo que efectivamente es, ya que debe transferir parte de su ganancia obtenida por renta informacional al regulador.

De este modelo puede concluirse lo siguiente:

a) Respecto de un escenario base, sin asimetrías de información, un regulador no benevolente no logra extraer rentas de ninguno de los operadores regulados.

b) Al existir asimetrías de información y un regulador no benevolente, el menú de tarifas logra resolver el problema de selección adversa inherente a tecnología $(\beta)$ y el de riesgo moral, en cuanto a inducción de esfuerzo óptimo por bajar costos (e). Sin embargo, como la función objetivo incluye un interés del regulador, es la empresa eficiente la que debe transferir al regulador parte de su renta informacional, que hace costoso para esta firma revelarse del tipo que es.

c) Si el operador eficiente considera alta la renta que le exige el regulador podría tener incentivos para revelarse como ineficiente, o bien, tal como señala Akerlof (1971), podría cumplirse la Ley de Gresham, donde el ineficiente puede llegar a desplazar parcial o totalmente al eficiente, generando por tanto un colapso del contrato regulatorio.

d) Con la renta que el regulador busca para sus propios intereses, la empresa ineficiente no ve afectado su menú de tarifas, por lo que le conviene actuar como tal sin buscar mayores ganancias en eficiencia.

e) Al verse afectado el menú de tarifas que se ofrece a la empresa eficiente y teniendo esta incentivos a salir del mercado o comportarse como una ineficiente, se pone en riesgo alcanzar una asignación óptima en el corto plazo y mejorar la eficiencia dinámica, lo que va en desmedro del bienestar de la sociedad en su conjunto. 


\section{Regulador no benevolente con un continuo de tipos}

Generalizando el modelo anterior con un continuo de tipos, la función objetivo que el regulador busca maximizar será la siguiente:

$$
\begin{gathered}
\max _{(q(\beta), e(\beta))} \int_{\underline{\beta}}^{\bar{\beta}}[V(q(\beta))-(1+\lambda)((\beta-e(\beta)) q(\beta)+\rho+\varphi(e(\beta))) \\
-\lambda(1-\alpha) U(\beta)] f(\beta) d \beta
\end{gathered}
$$

sujeto a:

$$
\frac{d U(\beta)}{d \beta}=-\frac{d \varphi(e(\beta))}{d e(\beta)}
$$

Sea el hamiltoniano

$$
\begin{gathered}
H=\int_{\underline{\beta}}^{\bar{\beta}}[V(q(\beta))-(1+\lambda)((\beta-e(\beta)) q(\beta)+\rho+\varphi(e(\beta)))-\lambda(1-\alpha) U(\beta)] f(\beta) \\
-\mu(\beta) \frac{d \varphi(e(\beta))}{d e(\beta)}
\end{gathered}
$$

donde $\frac{d H}{d U(\beta)}=-\frac{d \mu(\beta)}{d \beta}$

Se tiene entonces: $\lambda(1-\alpha) f(\beta)=\frac{d \mu(\beta)}{d \beta}$

$$
\lambda(1-\alpha) d F(\beta)=d \mu(\beta)
$$

Integrando por partes se tiene:

$$
\begin{gathered}
\int \lambda(1-\alpha) d F(\beta)=\int d \mu(\beta) \\
\mu(\beta)=\lambda(1-\alpha) F(\beta) \\
\frac{d H}{d q(\beta)}=\frac{d V(q(\beta))}{d q(\beta)}-(1+\lambda)(\beta-e(\beta))=0 \\
\frac{d V(q(\beta))}{d q(\beta)}=(1+\lambda)(\beta-e(\beta)) \\
\frac{d H}{d e(\beta)}=-(1+\lambda)\left(-q(\beta)+\frac{d \varphi(e(\beta))}{d e(\beta)}\right) f(\beta)-\mu(\beta) \frac{d^{2} \varphi(e(\beta))}{d e(\beta)^{2}}=0
\end{gathered}
$$


Se sabe que: $\mu(\beta)=\lambda(1-\alpha) F(\beta)$

reemplazando se tiene:

$$
\begin{gathered}
-\lambda(1-\alpha) F(\beta) \frac{d^{2} \varphi(e(\beta))}{d e(\beta)^{2}}=(1+\lambda)\left(-q(\beta)+\frac{d \varphi(e(\beta))}{d e(\beta)}\right) f(\beta) \\
\frac{d \varphi(e(\beta))}{d e(\beta)}=q(\beta)-\frac{\lambda}{1+\lambda}(1-\alpha) \frac{F(\beta)}{f(\beta)} \frac{d^{2} \varphi(e(\beta))}{d e(\beta)^{2}}
\end{gathered}
$$

Si se desea verificar el efecto de $\alpha$ en el nivel de esfuerzo se analiza la estática comparativa utilizando el teorema de la función implícita a partir de la cual se obtiene lo siguiente:

$$
\frac{d \varphi(e(\beta))}{d e(\beta)}-q(\beta)+\frac{\lambda}{1+\lambda}(1-\alpha) \frac{F(\beta)}{f(\beta)} \frac{d^{2} \varphi(e(\beta))}{d e(\beta)^{2}}=0
$$

Por tanto:

$$
\frac{d e(\beta)}{d \alpha}=\frac{\frac{\lambda}{1+\lambda} \frac{F(\beta)}{f(\beta)} \frac{d^{2} \varphi(e(\beta))}{d e(\beta)^{2}}}{\frac{d^{2} \varphi(e(\beta))}{d e(\beta)^{2}}+\frac{\lambda}{1+\lambda}(1-\alpha) \frac{F(\beta)}{f(\beta)} \frac{d^{3} \varphi(e(\beta))}{d e(\beta)^{3}}}>0
$$

El resultado al que se llega confirma lo encontrado para el caso de dos tipos, por lo que las implicancias en las tarifas son totalmente equivalentes a las analizadas en el caso anterior, es decir, cuando el regulador desea extraer rentas a alguno de los operadores eso se verá traducido en la presencia de una tarifa que no es óptima para el operador eficiente, generando finalmente costos sociales para los consumidores que hacen uso del bien o servicio regulado.

El modelo de regulador no benevolente y múltiples tipos de agentes permite concluir lo siguiente:

a) Con un regulador no benevolente y asimetría de información, la extracción de renta por parte del regulador hace que sea costoso para la firma eficiente revelarse del tipo que es, por lo que eventualmente si esta renta que extrae el regulador llegara a ser muy alta este operador podría declararse como ineficiente o bien salir del mercado. En ambos casos se cumple la Ley de Gresham provocando un colapso del contrato regulatorio.

b) La empresa ineficiente al no tener un efecto de $\alpha$ sobre su tarifa, no tiene incentivos a obtener mayores ganancias en eficiencia, generándose un costo en cuanto al nivel de eficiencia dinámica dentro de este mercado. 


\section{COMPARACION DE ESCENARIOS}

El modelo de regulador no benevolente con asimetría de información, donde el regulador pretende extraer rentas para su propio interés, muestra resultados no óptimos en el diseño de los menús de tarifas que eligen los operadores que ingresan a un mercado regulado. Los resultados a los que se arriban en materia de contrato regulatorio, a partir de cada modelo desarrollado, se resumen en la tabla siguiente.

\begin{tabular}{|c|c|c|}
\hline Escenario & Función objetivo & Contratos regulatorios \\
\hline $\begin{array}{l}\text { Regulador } \\
\text { benevolente } \\
\text { (información } \\
\text { simétrica) }\end{array}$ & $\begin{array}{c}\max _{(U, q, e)} W=V(q)-(1+\lambda)[(\beta-e) q+\rho \\
+\varphi(e)]-\lambda U\end{array}$ & $\begin{array}{l}\text { Tarifa óptima de primer mejor } \\
\qquad t=C^{*} \\
\text { donde } \\
\qquad C^{*}=\text { costo marginal }\end{array}$ \\
\hline $\begin{array}{l}\text { Regulador } \\
\text { benevolente } \\
\text { (información } \\
\text { asimétrica) }\end{array}$ & $\begin{array}{c}\max _{(\bar{q}, \underline{q}, \bar{e}, \underline{e})} W=v[V(\underline{q})-(1+\lambda)((\underline{\beta}-\underline{e}) \underline{q}+ \\
\rho+\varphi(\underline{e}))-\lambda \phi(\bar{e})]+(1-v)[V(\bar{q})- \\
(1+\lambda)((\bar{\beta}-\bar{e}) \bar{q}+\rho+\varphi(\bar{e}))]\end{array}$ & $\begin{array}{l}\text { Empresa eficiente: } \\
\qquad t^{*}(\underline{\beta})=\varphi(\underline{e})+\phi(\bar{e}) \\
\text { Empresa ineficiente: } \\
\qquad t^{*}(\bar{\beta})=\varphi(\bar{e})\end{array}$ \\
\hline $\begin{array}{l}\text { Regulador no } \\
\text { benevolente } \\
\text { (información } \\
\text { asimétrica) }\end{array}$ & $\begin{array}{c}\max _{(\bar{q}, \underline{q}, \bar{e}, \underline{e})} W=v[V(\underline{q})-(1+\lambda)((\underline{\beta}-\underline{e}) \underline{q}+ \\
\rho+\varphi(\underline{e}))-\lambda(1-\alpha) \phi(\bar{e})]+(1-v) \\
{[V(\bar{q})-(1+\lambda)((\bar{\beta}-\bar{e}) \bar{q}+\rho+\varphi(\bar{e}))]}\end{array}$ & $\begin{array}{l}\text { Empresa eficiente: } \\
\qquad t^{*}(\underline{\beta})=\varphi(\underline{e})+\phi(\bar{e}) \\
\text { Con: } G=\lambda \alpha(\phi(\bar{e})) . \\
\text { donde: } \frac{d t}{d \alpha}=\frac{d \phi(\bar{e})}{d \bar{e}} \frac{d \bar{e}}{d \alpha}>0 \\
\text { Empresa ineficiente: } \\
\qquad t^{*}(\bar{\beta})=\varphi(\bar{e})\end{array}$ \\
\hline
\end{tabular}

De dicha tabla se concluye que en la medida que los reguladores son benevolentes, ya sea que enfrenten asimetrías de información o no, el menú de tarifas logra que las firmas se revelen como lo que son y se maximiza el nivel de bienestar social. Si bien con asimetrías de información se debe incurrir en una renta informacional para motivar al eficiente a que participe en el mercado, el mecanismo plasmado en la tarifa logra resolver el problema de selección adversa y de riesgo moral.

En un modelo de asimetrías de información con un regulador no benevolente que pretende sacar rentas de alguno de los operadores regulados, el resultado de la regulación económica se traduce en el diseño de tarifas que resuelven los problemas de asimetría de información pero que introducen un costo a la empresa eficiente, la que puede dejar de tener incentivos para participar dentro del contrato regulatorio y 
para operar de forma eficiente, ocasionando finalmente un costo social elevado para los consumidores que forman parte del mercado regulado.

Escenarios como el anterior llaman a la reflexión respecto de situaciones en las que la regulación debería privilegiar, en todos los casos, la maximización del bienestar de la sociedad, ya que cuando por algún motivo, político, social o económico, el regulador busca la extracción de rentas de los agentes regulados, se pueden generar notorias distorsiones en el funcionamiento de los contratos regulatorios. Al respecto, si se asume que los agentes son de diversos tipos, con funciones de costo diferentes, se puede terminar desplazando a la empresa eficiente en la medida que esta no esté dispuesta a realizar la transferencia al regulador. Este aspecto, ya presentado por Akerlof (op. cit.), origina que el contrato regulatorio colapse al no poder corregirse adecuadamente el problema de información asimétrica llevando a que en el mercado se desincentive la eficiencia generando por tanto altos gastos a los consumidores finales.

\section{CONCLUSIONES}

De la revisión realizada se pueden arribar a las siguientes conclusiones:

$\checkmark \quad$ Cuando un mercado es regulado por un regulador benevolente que además posee información perfecta acerca de la tecnología de las empresas así como del nivel de esfuerzo que las firmas realizan para bajar sus costos, se logra fijar una tarifa eficiente que hace máximo el nivel de bienestar de la sociedad. En general, bajo este escenario se logra alcanzar niveles de eficiencia asignativa que son equivalentes a la formulación de contratos de alto poder, donde los agentes tienen incentivos a realizar esfuerzos óptimos que permiten alcanzar niveles de costo de prestación del servicio más bajos.

$\checkmark$ Bajo un escenario de un regulador benevolente que enfrenta asimetrías de información, el diseño de un mecanismo óptimo, traducido en la tarifa, logra que los agentes se autorrevelen como lo que efectivamente son, es decir, los eficientes se declararán como tales así como los ineficientes. Lo anterior es factible en la medida que la función objetivo del regulador se maximiza sujeto a la restricción de compatibilidad de incentivos y de racionalidad individual. Se encuentra que bajo un escenario de información asimétrica el regulador debe incurrir en un costo (renta informacional) por obtener información de quién es la empresa más eficiente, lo que finalmente se traduce en el logro de tarifas óptimas, que permiten alcanzar un equilibrio separador.

$\checkmark \quad$ Un regulador no benevolente, que enfrenta diferentes tipos de agentes y busca extraer rentas de algunos de ellos, solo puede obtener ventajas cuando están presentes las asimetrías de información. Lo anterior es cierto porque con información perfecta, no solo el regulador ve el esfuerzo y conoce la función de producción de las firmas, sino que a su vez, son los operadores los que conocen dicha información. Es claro que cualquier tipo de operador (eficiente o ineficiente) que posea información perfecta no aceptará que el regulador quiera cobrarle rentas por algo que él ya conoce, por lo que en un escenario donde se quiera obtener alguna ventaja por 
parte de los operadores, este puede optar por no ingresar a este mercado que presentaría una regulación asimétrica.

El diseño del contrato regulatorio, de un regulador no benevolente con asimetrías de información, muestra que el menú de tarifas resuelve el problema de la información asimétrica en cuanto a tecnología y esfuerzo óptimo a ser realizado por las firmas. Se muestra que la tarifa ofrecida al operador eficiente incluye una renta informacional que lo motiva a revelarse como tal, sin embargo, ya que el regulador pretende llevarse, para su propio beneficio, una parte de dicha renta informacional, genera que la firma eficiente tenga incentivo a no participar del contrato, o bien a comportarse como una firma ineficiente porque revelarse como lo que es tiene un costo. Si la transferencia exigida por el regulador llegara a ser muy alta ( $\alpha$ tendiendo a 1$)$ el operador ineficiente podría quedar indiferente ante el contrato del eficiente, porque se le exige un nivel de esfuerzo demasiado alto generando un colapso del contrato regulatorio formulado, llevando a evaluar en términos prácticos la pertinencia de mantener un esquema regulatorio que no logra cumplir con los objetivos de eficiencia asignativa y máximo nivel de bienestar para la sociedad.

\section{BIBLIOGRAFIA}

AKERLOF, G. (1971). "The market for "lemons": Quality, Uncertainty and the Market Mechanism", The Quarterly Journal of Economics 84 (3), pp. 488-500.

ARMSTRONG, M. y D. SAPPINGTON (2005). Recent Developments in the Theory of Regulation. University of Florida, Florida.

BARON, D. y R. MYERSON (1982). "Regulating a Monopolist with Unknown Costs", Econometrica 50 (4), pp. 911- 930.

BAUMOL, W.J., J.C. PANZAR y R.D. WILLIG (1982). Contestable Markets and the theory of industry structure. Harcourt Brace Jovanovitch, New York.

BONBRIGHT, J.C. (1961). Principles of Public Utility Rates. Columbia University Press.

JEHLE, G. y P. RENY (2011). Advanced Microeconomic Theory, Third Edition. Prentice Hall.

LAFFONT, J.J. y J. TIROLE (1999). A Theory of incentives in procurement and regulation. MIT, Cambridge, Massachusetts.

LAFFONT, J.J. (2005). Regulation and development. University Press, Cambridge.

PELTZMAN, S. (1976). "Toward a more General Theory of Regulation”, Journal of Law and Economics 19 (2), pp. 211-240.

PIGOU, A.C. (1932). The Economics of Welfare. Macmillan and Co., London.

POSNER, R. (1974). "Theories of Economic Regulation", The Bell Journal of Economics and Management Science 5 (2), pp. 335-358.

ROSS, S. (1973). "The Economic theory of agency: The principal's problem", American Economic Association 63 (2), pp. 134-139.

SPENCE, M. (1973). "Job Market Signaling”, The quaterly journal of economics 87 (3), MIT Press.

SHY, O. (1995). Industrial Organization, Theory and applications. The MIT Press, Cambridge, Massachusetts.

STIGLER, G. (1971). "The theory of Economic Regulation", The Bell Journal of economics and management science 2 (1), pp. 3-21.

VICKERS, J. y G. YARROW (1988). Privatization: An Economic Analysis. The MIT Press, Cambridge, Massachusetts. 
